ISSN 1997-5902

\title{
Influence de la fertilisation à base des coques de cacao sur les paramètres chimiques d'un ferralsol et sur la croissance du maïs (Zea mays L.) à Oumé, Côte d'Ivoire.
}

\begin{abstract}
ABOBI Akré Hébert Damien ${ }^{1}$, ANGUI Tehua Kouassi Pascal ${ }^{2}$, KOUADIO Yatty Justin 1
1. Université Nangui Abrogoua. Unité de Formation et de Recherche des Sciences de la Nature (UFR-SN) Laboratoire de Biologie et d'Amélioration des Productions Végétales (LBAPV). 02 BP 801 Cl-Abidjan 02 (Côte d'Ivoire). hebertabobi@gmail.com ou djekdanhomath@yahoo.fr

2. Université Nangui Abrogoua. Unité de Formation et de Recherche des Sciences et Gestion de l'Eau (UFR-SGE) Laboratoire de Géosciences et Environnement. 02 BP 801 Cl-Abidjan 02 (Côte d'Ivoire). pascalangui@hotmail.com
\end{abstract}

Original submitted in on $9^{\text {th }}$ September 2014. Published online at www.m.elewa.org on $31^{\text {st }}$ October 2014. http://dx.doi.org/10.4314/jab.v82i1.4

\section{RESUME}

Objectifs : Evaluer les effets des broyats de coques de cacao dans la fertilisation des ferralsols d'Oumé pour une meilleure croissance du maïs.

Méthodologie et résultats : L'essai a été installé suivant un dispositif en blocs de Fischer comportant trois répétitions. Les effets de trois doses de broyats de coques $0 ; 1,39$ et 2,78 $\mathrm{g}$ pour $100 \mathrm{~g}$ de sol sec, correspondant à (0 (D0), 5 (D5) et $10 \mathrm{~g}$ carbone.kg-1 (D10), ont été évalués sur la fertilité du sol et la croissance du maïs. La minéralisation du carbone $(C)$, les paramètres végétatifs du maïs et les paramètres chimiques du sol ont été observés à des dates régulières. La minéralisation du $\mathrm{C}$ a augmenté de $64,40 \%$, avec D5, alors qu'elle a baissé de 70,43\%, avec D10, par rapport à D0. La dose D5 a permis une augmentation de la biomasse aérienne de 21,53 et $66,75 \%$ par rapport à D0. Avec D10, cette biomasse a baissé de $29,69 \%$, au $1^{\mathrm{er}}$ cycle, tandis qu'elle a augmenté de $16,94 \%$, au $2^{\mathrm{e}}$ cycle. Les paramètres chimiques des sols ont été améliorés de façon significative et proportionnelle avec les coques de cacao ( $p$ $<0,001)$.

Conclusion et application: Les coques de cacao constituent une source de matière organique et de nutriments pour l'amélioration de la fertilité du sol et la croissance du maïs. Par conséquent, elles pourraient être utilisées dans la fertilisation des ferralsols de la zone d'Oumé. La pression agricole étant forte et l'utilisation des engrais minéraux fort limitée par leurs coûts prohibitifs, leur intégration dans la gestion de la fertilité du sol pourrait être recommandée en vue d'une agriculture durable.

Mots clés : Coques de cacao, fertilité du sol, ferralsols, maïs, Côte d'Ivoire. 


\section{Abobi et al. J. Appl. Biosci. 2014. Influence de la fertilisation à base des coques de cacao sur les paramètres chimiques d'un ferralsol et sur la croissance du maïs à Oumé Cote d'Ivoire}

Influence of fertilization based on cocoa hulls on a ferralsol chemical parameters and on maize (Zea mays $L$.) growth

\section{ABSTRAT}

Objectives: The aim of this study was to evaluate effects of cocoa hulls in restoring ferralsol fertility in Oume area (Mid West of Côte d'Ivoire) to increase the growth of maize. Methodology and Results: The trial was installed following a Fischer blocks design with three replications. The effects of three doses of cocoa

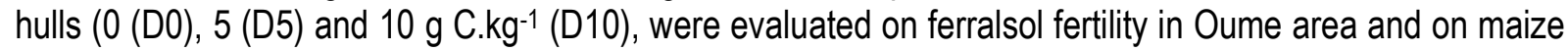
growth. Carbon (C) mineralization, soil chemical parameters and growth parameters of maize were monitored. Carbon mineralization increased by $64,4 \%$ with D5 while it decreased by $70,4 \%$ with D10, compares to D0. Although, cocoa hulls impact significantly maize height, biomass production was significantly increased particularly with D5. Values of soil chemical characteristics recorded at maize harvesting were significantly increased and the rate of improvement was proportional to the dose of $\mathrm{C}$ $(p<0,001)$. Conclusion and Application: the study showed that cocoa hulls can be used to improve soil chemical parameters and maize crop. Cocoa hulls application may be a promising mean for the rehabilitation of soil fertility in Oumé area. However, the quantity must be controlled.

Keywords: Coco hulls, soil fertility, ferralsols, maize, Côte d'Ivoire.

\section{INTRODUCTION}

La pérennisation de la culture du maïs, céréale la plus cultivée après le riz et le blé et aliment de base de nombreuses populations en Côte d'Ivoire (Akanvou et al., 2006), est liée au maintien de la fertilité du sol (Rouanet, 1984). Or, la forte pression sur les terres agricoles occasionne une baisse significative de la fertilité du sol et des rendements des cultures (Bado, 2002; Boga, 2007). Les engrais minéraux, dont on a eu recours pour maintenir le niveau des éléments nutritifs, en raison de leur plus grande efficience agronomique (Useni et al., 2013), sont presqu'inaccessibles aux agriculteurs, à cause de leurs coûts prohibitifs et leur indisponibilité en milieu rural (Somé et al., 2007). Leur utilisation continue et exclusive entraîne une baisse de la productivité de sols (Useni et al., 2013) et une diminution des rendements (Boli et Roose, 2000), outre les effets négatifs sur l'environnement (Bado, 2002). Le rôle

\section{MATERIELS ET METHODES}

Site Les sols des cultures vivrières (CV) du site du projet «Conservation and Sustainable Management of Below Ground Biodiversity) (CSM-BGBD), ont été utilisés. Ce site est localisé à Oumé, dans le village de Goulikao (Fig. 1), de coordonnées géographiques $5^{\circ} 31^{\prime} \mathrm{W}, 6^{\circ} 17^{\prime} \mathrm{N}$ et $200 \mathrm{~m}$ ). Le climat est de type subéquatorial « attiéen » avec une végétation dense et important des fertilisants organiques, sur diverses propriétés du sol, est largement reconnu, permettant de justifier leur utilisation (Koulibaly et al., 2009). Quelques travaux ont été publiés sur l'effet des coques de cacao dans la fertilisation des sols (Moyen-Jesu, 2007 ; Djéké et al., 2011). Les coques de cacao constituent une source importante de potassium pour le maïs (AduDapaah et al., 1994),une source de matières organiques et un réservoir d'éléments chimiques (Djéké et al., 2011). Leur disponibilité en Côte d'Ivoire, 1er producteur mondial de cacao (Lepoitevin et al., 2003), milite en faveur de leur valorisation agricole en vue de leur utilisation pour améliorer la fertilité des sols. L'objectif de ce travail est d'évaluer les effets des doses croissantes de résidus des coques de cacao sur les paramètres chimiques des ferralsols de la zone d'Oumé et sur la croissance du maïs.

semi-décidue. La pluviométrie moyenne est de 1300 $\mathrm{mm} / \mathrm{an}$ avec une température moyenne de $26^{\circ} \mathrm{C}$ et une humidité moyenne de $85 \%$ (Bongoua, 2002). Les surfaces affectées aux cultures vivrières sont dégradées (Angui et al., 2005). Les sols d'Oumé sont des ferralsols (FAO, 2006), avec un $\mathrm{pH}$ bas $(5,30)$, une texture sablo-argileuse (21\% d'argile), une faible 


\section{Abobi et al. J. Appl. Biosci. 2014. Influence de la fertilisation à base des coques de cacao sur les paramètres chimiques d'un ferralsol et sur la croissance du maïs à Oumé Cote d'Ivoire}

teneur en carbone organique $(\mathrm{C} / \mathrm{N}=10,47)$ et une capacité d'échange cationique faible $\left(7,22 \mathrm{cmol} \mathrm{kg}^{-1}\right)$.

Matériel organique: Le matériel organique est constitué de coques de cacao (Theobroma cacao), ramassés dans les plantations de cacao du Centre National de Recherche Agronomique (CNRA-Côte d'Ivoire). Ces coques ont été séchées à l'air libre pendant 7 jours, ensuite broyées et tamisées $(\varnothing<2$ $\mathrm{mm})$. Elles présentent des rapports $\mathrm{C} / \mathrm{N}$ et $\mathrm{C} / \mathrm{P}$ de
25,71 et 400 , respectivement. Leurs compositions, déterminées à partir de la matière sèche, indiquent 36 $\%$ de $\mathrm{C} 1,4 \%$ de $\mathrm{N} 0,09 \%$ de P $0,55 \%$ de $\mathrm{Ca}^{2} \mathrm{O}, 42 \%$ de $\mathrm{Mg}^{2+}$ et 3,39\% de $\mathrm{K}^{+}$.

Matériel végétal : Le matériel végétal est la variété de maïs "Rouge de Ferké EV8717" ayant un cycle relativement court (90 à $120 \mathrm{j}$ ), et un rendement potentiel de 6 à 7 tha. Les grains ont un poids moyen de $390 \mathrm{mg}$.

(a)
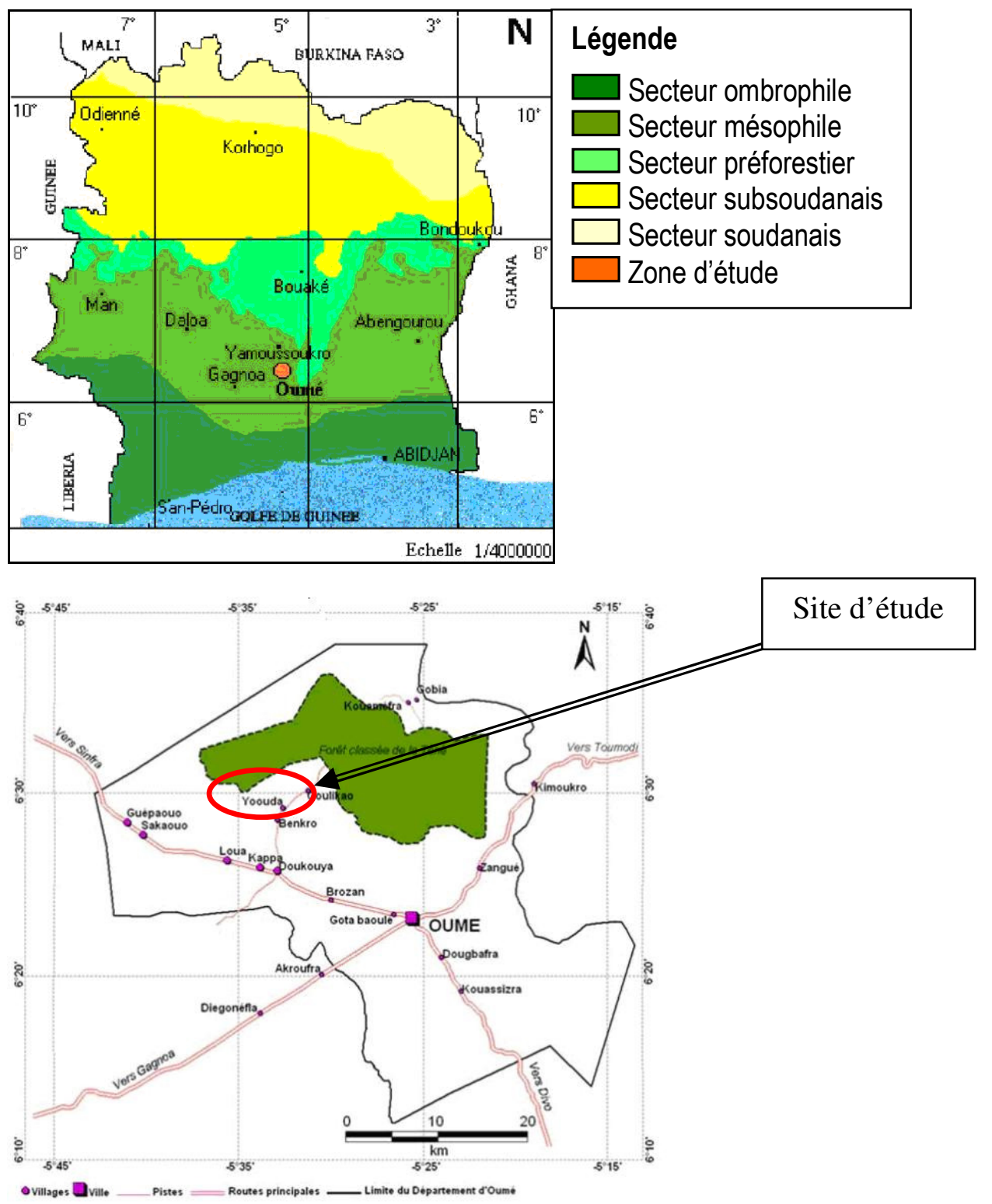

(b)

Figure 1 : Localisation du site d'Oumé : (a) ville d'Oumé, (b) village de Goulikao (Bongoua, 2002).

Dispositif expérimental et conduite des essais : Les essais ont été conduits selon un dispositif expérimental en blocs de Fischer comportant trois répétitions. Les traitements suivants : sol de CV sans apport (D0), sol de CV + 5 gC. $\mathrm{kg}^{-1}$ (D5) et sol de CV + 10 gC. $\mathrm{kg}^{-1}$ (D10) ont été étudiés. L'essai d'incubation a été conduit au 


\section{Abobi et al. J. Appl. Biosci. 2014. Influence de la fertilisation à base des coques de cacao sur les paramètres chimiques d'un ferralsol et sur la croissance du maïs à Oumé Cote d'Ivoire}

laboratoire de Biologie et d'Amélioration des Productions Végétales de l'Université Nangui Abrogoua. Deux quantités de broyats de coques de cacao : 1,39 et $2,78 \mathrm{~g}$, correspondant respectivement à D5 et D10, ont été mélangées à $100 \mathrm{~g}$ de sol sec de $\mathrm{CV}$ et comparées à un témoin non amendé (D0). Les incubations ont été réalisées dans des bocaux de 11 , en conditions contrôlées, à une humidité proche de la capacité au champ ( $20 \%$ en humidité pondérale) et à une température constante $\left(28^{\circ} \mathrm{C} \pm 2\right)$. Les bocaux ont été fermés hermétiquement et gardés dans l'obscurité. L'humidité a été contrôlée constamment, par pesée, et ajustée au besoin. L'essai de culture du maïs a été mis en place, dans les conditions semi-contrôlées, sur le site expérimental de l'Université Nangui Abrogoua. Les deux quantités de broyats de coques de cacao déterminées (1,39 et 2,78 g), ont été multipliées par 10, soit: 13,9 et $27,8 \mathrm{~g}$, et apportées à $1000 \mathrm{~g}$ de sol sec de $\mathrm{CV}$, dans des pots. Trois grains de maïs ont été semés dans chaque pot. Le démariage a été effectué à un seul plant par pot une semaine après semis. Deux cycles de maïs ont été réalisés pour tester le potentiel fertilisant des coques de cacao.

Minéralisation $\mathrm{du}$ carbone organique: La minéralisation du $\mathrm{C}$ a été mesurée par piégeage de $\mathrm{CO}_{2}$ dans la soude (Freijer and Bouten, 1991). Les quantités de $\mathrm{CO}_{2}$ ont été déterminées par titrage avec du $\mathrm{HCl} 0,1 \mathrm{M}$, en présence de phénolphtaléine $5 \%$, de façon régulière, jusqu'à $120 \mathrm{j}$ d'incubation. Ces quantités ( $\mathrm{mg} \mathrm{C}-\mathrm{CO}_{2} \mathrm{~kg}^{-1}$ de sol) ont été calculées, à l'aide de la formule suivante (Anderson and Ingram, 1993):

$Q\left(\mathrm{mg} \mathrm{C} \mathrm{kg}^{-1}\right)=\left[\left(\mathrm{V}_{\mathrm{bl}}-\mathrm{V}_{\text {éch }}\right) \times 2,2 \times 1000\right] / 100(1)$

\section{RESULTATS}

Minéralisation du carbone organique : Les teneurs cumulées en $C$ minéralisé, présentées sur la figure 1 , ont été significativement différentes $(p<0,001)$ à toutes les périodes d'incubations. Les plus fortes teneurs ont été obtenues avec la dose D5 et les plus faibles avec où

$\mathrm{V}_{\mathrm{bl}}=$ volume moyen de $\mathrm{HCl}$ pour le contrôle,

$V_{\text {éch }}=$ volume de $\mathrm{HCl}$ pour l'échantillon,

$100=$ poids de sols $(\mathrm{g})$.

Paramètres de croissance du maïs : La hauteur des plants de maïs a été mesurée tous les 3 jours, du $11 \mathrm{e}$ au $56 \mathrm{e}$ jour après semis (JAS). Les biomasses aérienne et souterraine des plants ont été déterminées au $56^{\mathrm{e}}$ jas, après séchage à $60^{\circ} \mathrm{C}$ dans une étuve biologique pendant 72 heures. L'indice de vigueur (IV), caractère important du développement des plants (Berchoux et Lecoustre, 1986), a été déterminé, tous les 3 jours, du $11^{\mathrm{e}}$ au $56^{\mathrm{e}}$ jas, selon la formule suivante:

$$
\text { IV } \left.=\log \left[\left(C^{2} \times H\right) / 4 \pi\right)\right](2)
$$

où $\mathrm{C}=$ circonférence au collet, $\mathrm{H}=$ hauteur.

Paramètres chimiques du sol: A la fin de l'essai de culture, les mesures de $\mathrm{pH}$ ont été effectuées dans une suspension de sol dans l'eau et dans une solution normale de chlorure de potassium $(\mathrm{KCl}) 1 \mathrm{M}$, à l'aide d'un $\mathrm{pH}$-mètre, dans un rapport sol/solution 1/2,5. La méthode à l'acétate d'ammonium $1 \mathrm{M}$ à $\mathrm{pH} 7$ (Anderson and Ingram, 1993) a été utilisée pour déterminer la capacité d'échange de cations (CEC) et les teneurs en bases échangeables $\left(\mathrm{Ca}^{2+}, \mathrm{Mg}^{2+}, \mathrm{K}^{+}\right)$.

Analyse statistique des données: Les données recueillies ont été soumises à une analyse de variance (ANOVA), avec test post hoc (test de Newman-Keuls) pour la comparaison des moyennes, lorsque des différences significatives ont été observées, au seuil de probabilité de $5 \%$. Ces tests ont été réalisés à l'aide du logiciel Statistica 7.1.

D10 (Fig. 2). A la fin des incubations, D5 a permis d'avoir une augmentation importante de 64,40\%, par rapport aux sols non amendés (D0), tandis que D10 a occasionné une baisse de $70,43 \%$. 


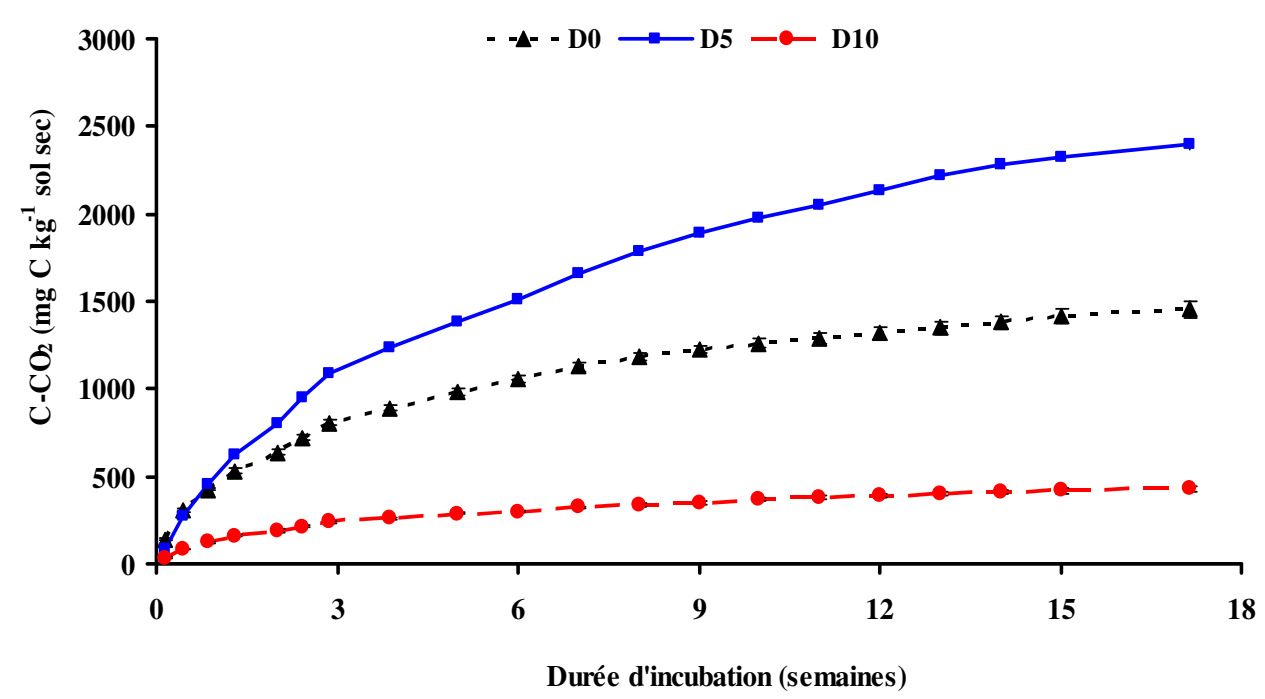

Figure 2 : Evolution des quantités cumulées de $\mathrm{C}-\mathrm{CO}_{2}$ dégagé en fonction des doses de $\mathrm{C}$ et du temps d'incubation.

Hauteur des plants : Au 1er cycle, les différences entre les hauteurs des plants ont été significatives aux 14 e, $21^{\mathrm{e}}$ et $28^{\mathrm{e}}$ jas $(0,006 \leq p \leq 0,025)$, tandis qu'au $2^{\mathrm{e}}$ cycle, les différences ont été significatives à toutes les dates de mesure $(0,002 \leq p \leq 0,044)$. La figure 3 montre qu'au 1 er cycle, les plus petites hauteurs ont été obtenues avec D10, alors que les effets des doses D0 et D5 ont été similaires. Au $2^{\mathrm{e}}$ cycle, les hauteurs des plants des sols ayant reçu D5, ont été les plus fortes, avec des accroissements de 18,28 à 30,83 \% par rapport à D0 (Fig. 4). En revanche, avec D10, les hauteurs ont été les plus faibles, pendant les 35 premiers JAS, en diminuant de 11,55 à $18,71 \%$ par rapport à celles obtenues avec D0. Aux 49e et 56e JAS, les hauteurs des plants des sols ayant reçu D5 ont été inférieures de 5,35 et $9,01 \%$ à celles des plants des sols fertilisés à la dose D10 (Fig. 4).

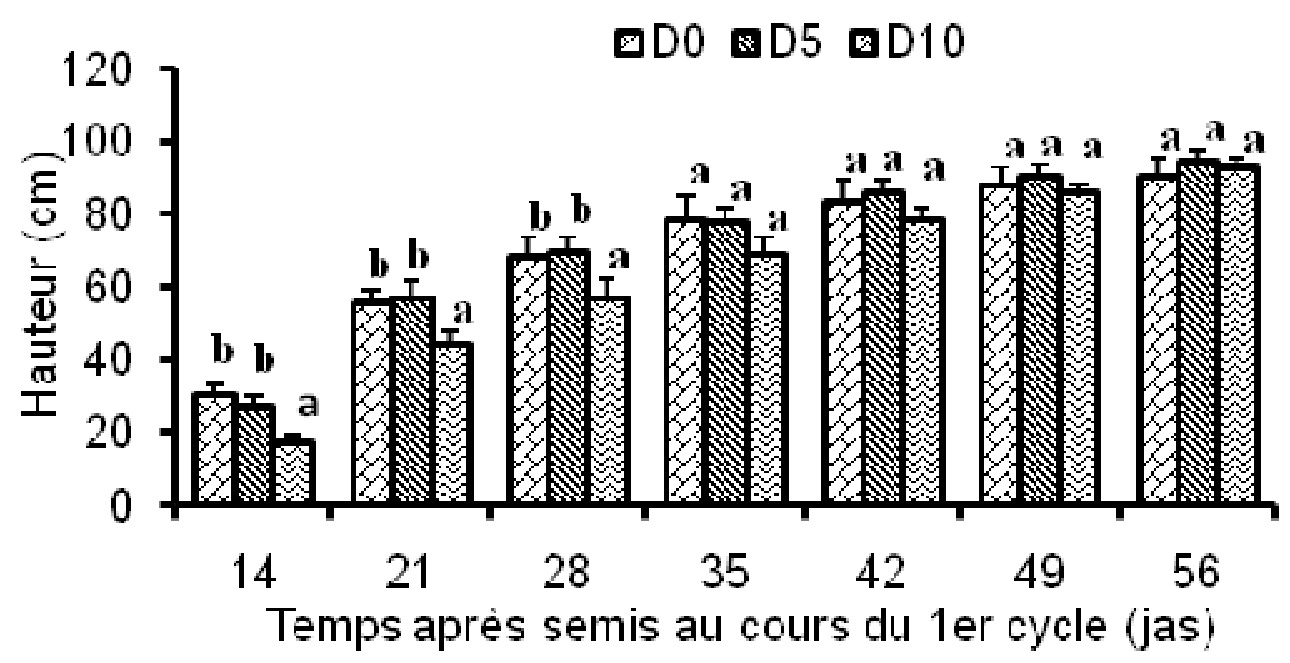

Figure 3 : Evolution des hauteurs des plants de maïs, en fonction des doses de carbone et du temps après semis, au cours du 1 er cycle. 


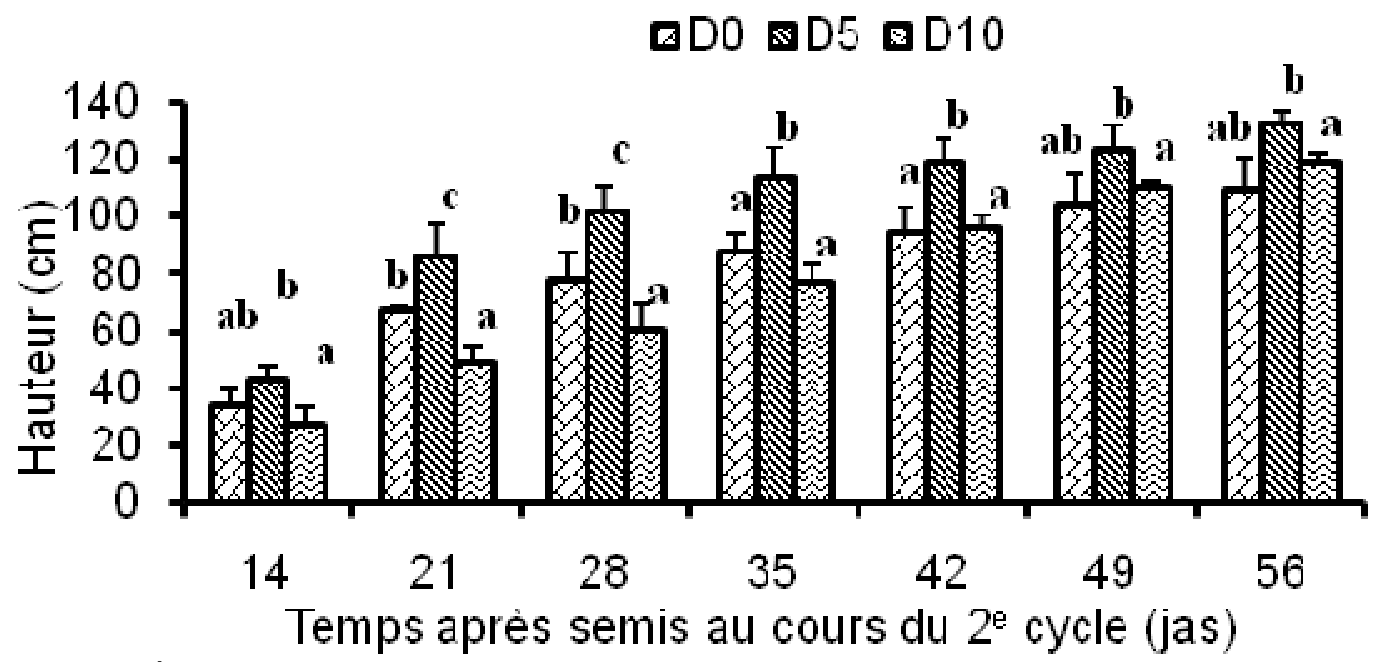

Figure 4 : Évolution des hauteurs des plants de maïs, en fonction des doses de carbone et du temps après semis, au cours du $2^{\mathrm{e}}$ cycle.

Indice de vigueur. Les différences entre les indices de vigueur des plants, au 1er cycle, ont été hautement significatives au 14 e JAS $(p=0,006)$, mais non significatives du $21^{\mathrm{e}}$ au $56^{\mathrm{e}}$ JAS $(0,25 \leq p \leq 0,66)$. Au $14 \mathrm{e} \mathrm{JAS}$, les indices obtenus avec D10 ont été inférieurs de $12,04 \%$ à ceux enregistrés avec D0; alors que ces derniers ont été similaires à ceux obtenus avec D5 (Fig. 5). Mais, la tendance montre que les indices obtenus avec la dose D5 ont été plus forts. En revanche, les indices de vigueur ont été significativement différents durant le 2 e cycle $(0,001 \leq$ $p \leq 0,044)$. Avec D5, les plants ont présenté les plus fortes valeurs, avec des accroissements de 5,70 à $10,74 \%$ par rapport à D0, parallèlement D0 et D10 ont produit des effets similaires (Fig. 6).

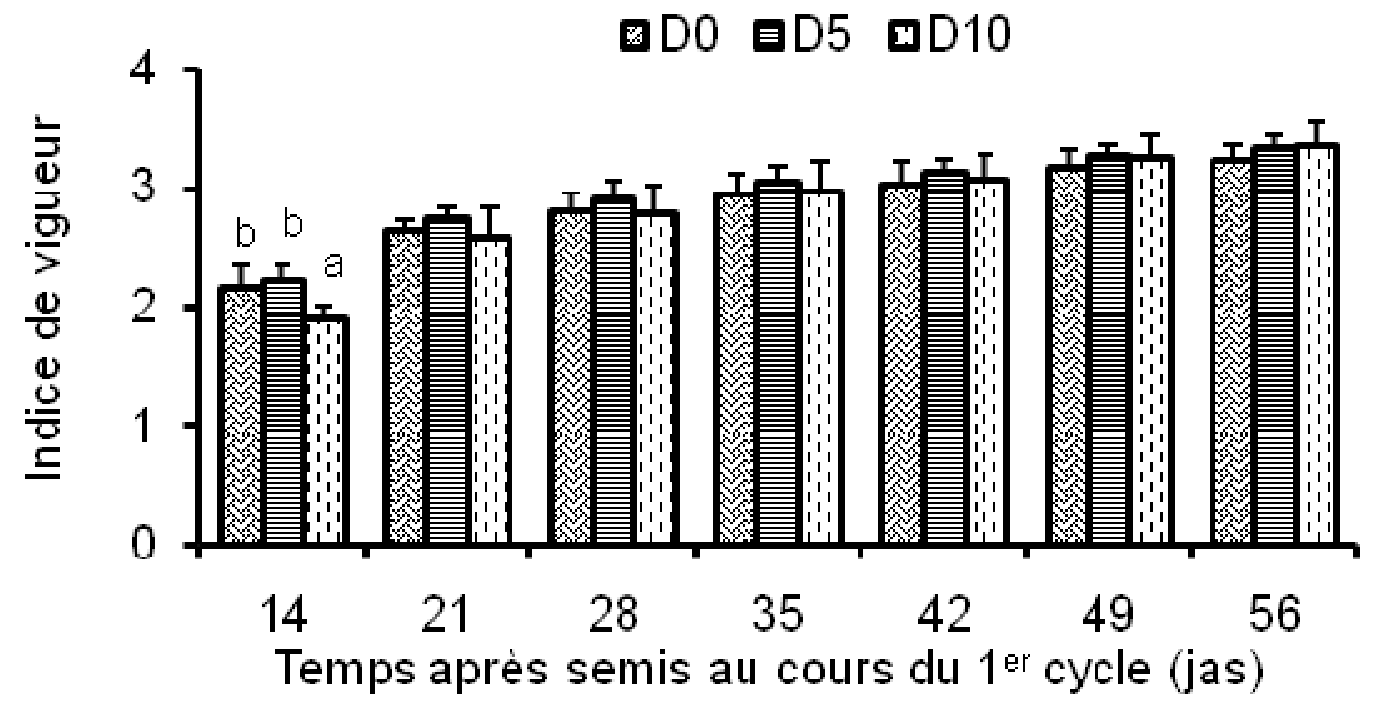

Figure 5 : Évolution des indices de vigueur moyens des plants de maïs, en fonction des doses de carbone et du temps après semis, au cours du 1er cycle. 


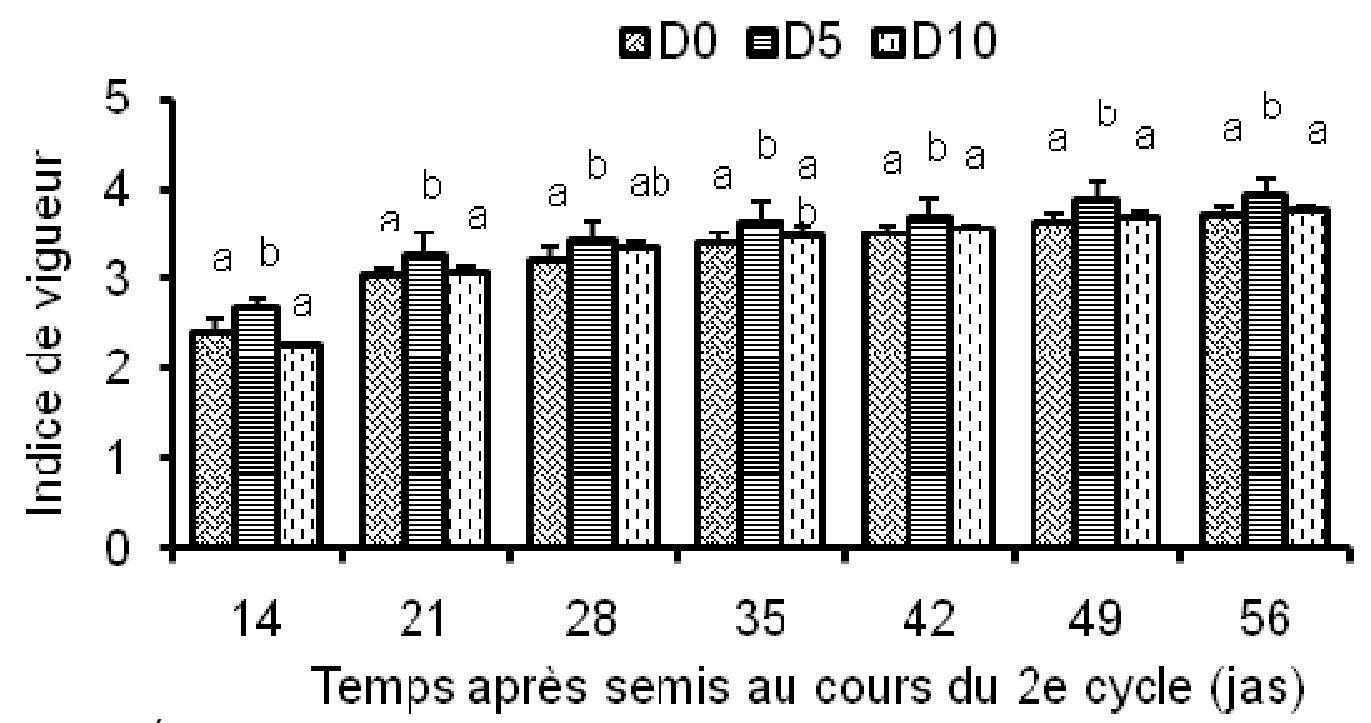

Figure 6 : Évolution des indices de vigueur moyens des plants de maïs, en fonction des doses de carbone et du temps après semis, au cours du $2^{\mathrm{e}}$ cycle.

Biomasses aérienne et souterraine. Les coques de cacao ont induit des différences hautement significatives $(0,001 \leq p \leq 0,003)$. Les plus fortes biomasses aériennes ont été obtenues avec D5, qui a permis, par rapport à $\mathrm{D} 0$, des augmentations respectives de 21 , et 66,73 p.c., aux 1 er et 2 e cycles. Les plus petites biomasses ont été enregistrées avec D10, au 1er cycle, et avec D0, au 2e cycle. Par rapport à D0, D10 a occasionné une perte de 29,69 p.c., au 1er cycle et une augmentation de 16,94 p.c., au 2 e cycle, (Fig. 7). Les biomasses souterraines des plants de maïs n'ont pas été significativement différentes $(p=$ 73,84), au 1er cycle. Toutefois, elles ont été proportionnelles aux quantités de coques de cacao
(Fig. 7). Les différences observées au $2 e$ cycle ont été hautement significatives $(p=0,002)$. L'apport des coques de cacao a permis d'avoir les plus fortes valeurs, elles ont été plus grandes avec D5 qu'avec D10, pour des augmentations respectives de 88,73 p.c., contre 77,01 p.c. (Fig. 8).

Paramètres chimiques du sol pH des sols. À la fin des deux cycles de culture de maïs, les $\mathrm{pH}$ des sols ont présenté des différences significatives $(p \leq 0,018)$. Les $\mathrm{pH}$ des sols fertilisés ont été significativement supérieurs à ceux des sols non fertilisés (Fig. 9). Les doses D5 et D10 ont produit des effets similaires sur les $\mathrm{pH}$ qui ont connu des augmentations de 0,3 unité par rapport à ceux des sols non fertilisés (D0).

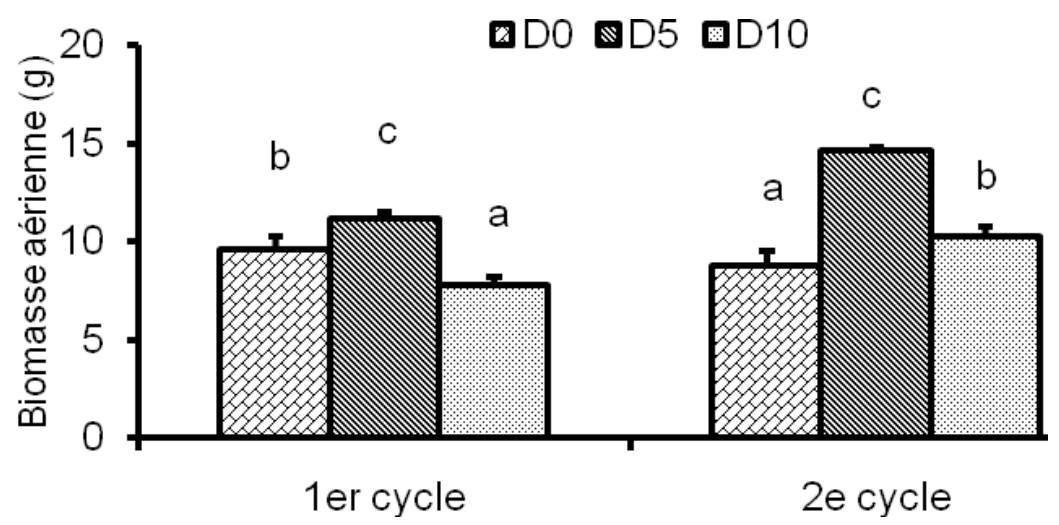

Figure 7 : Biomasses aériennes moyennes des plants de maïs ( $\left.\mathrm{g} \mathrm{plant}^{-1}\right)$ obtenues en fonction des doses de carbone à la fin de l'essai de culture de maïs. 


\section{Abobi et al. J. Appl. Biosci. 2014. Influence de la fertilisation à base des coques de cacao sur les paramètres chimiques d'un ferralsol et sur la croissance du maïs à Oumé Cote d'Ivoire}

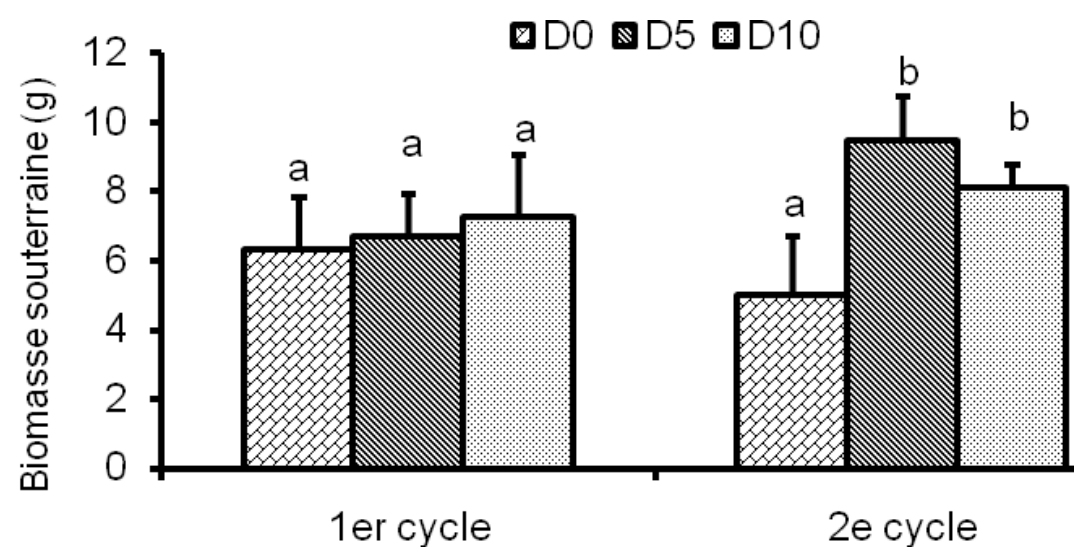

Figure 8 : Biomasses souterraines moyennes des plants de maïs (g plant-1) obtenues en fonction des doses de carbone à la fin de l'essai de culture de maïs.

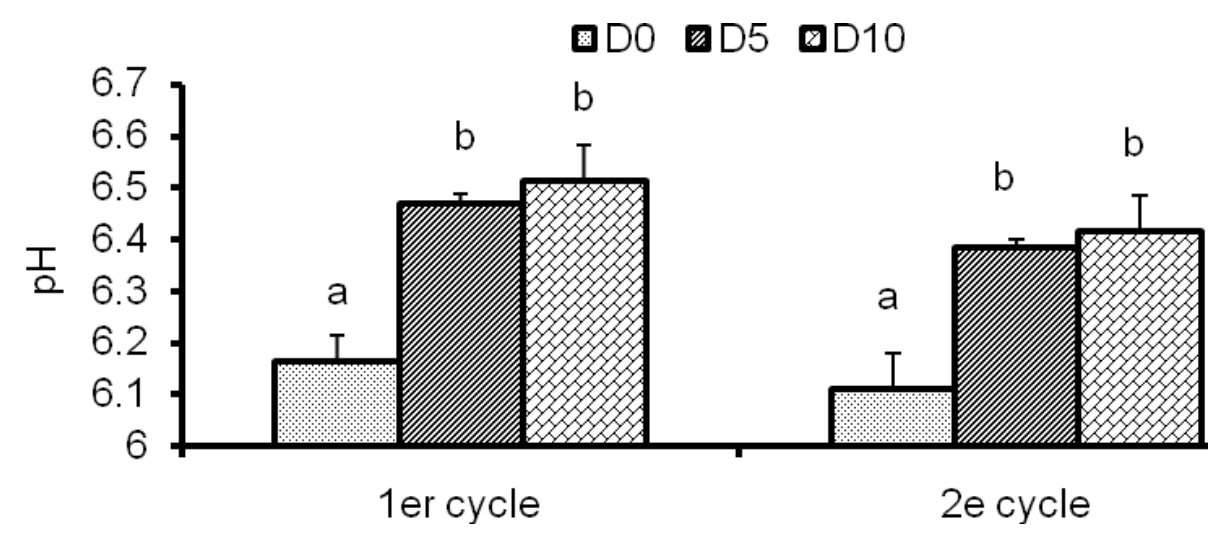

Figure 9: pH des sols, en fonction des doses de C, à la fin de l'essai de culture de maïs.

CEC des sols. Après les deux cycles de culture, les différences observées entre les CEC ont été hautement significatives $(p \leq 0,001)$. Les CEC ont été proportionnelles aux doses de C (Fig. 10). Les augmentations respectives de 4,63 et 4,99\%, avec la dose D5, et de 7,96 et 10,56\%, avec la dose D10, ont été obtenues par rapport à $\mathrm{D} 0$, aux $1^{\text {er }}$ et $2^{\mathrm{e}}$ cycles de culture de maïs.

Teneurs en calcium. Les coques de cacao ont produit des effets positifs sur les teneurs en $\mathrm{Ca}^{2+}$ des sols, au cours des deux cycles de maïs; les différences entre ces teneurs ont été hautement significatives $(p \leq 0,01)$. Les valeurs ont été proportionnelles aux doses de $C$ (Fig. 11). Comparées aux sols non fertilisés (D0), D5 et D10 ont permis d'obtenir des accroissements respectifs de 71,14 et $40,89 \%$, contre 136,63 et $127,78 \%$, aux 1 er et 2 e cycles.
Teneurs en magnésium: Les teneurs des sols en $\mathrm{Mg}^{2+}$ ont connu des différences très hautement significatives, à la fin des deux cycles de culture de maïs $(p<0,001)$. Ces teneurs ont été fonction des doses de carbone, celles obtenues avec les doses D5 et D10 ont été respectivement 2 et 3 fois supérieures à celles de D0 (Fig. 12)

Teneur en potassium : Le test de Newman Keul a révélé des différences hautement significatives $(p<$ 0,001 ), entre les teneurs en $\mathrm{K}^{+}$, après huit semaines de culture. Les doses D5 et D10, des teneurs en $\mathrm{K}^{+}$qui sont, respectivement, 2 et 3 fois supérieures à celles des sols non fertilisés (D0) (Fig. 13). Les accroissements obtenus avec D5 ont été de 93,72 et $99,12 \%$, contre 171,13 et $183,27 \%$, par rapport à D0 respectivement, aux $1^{\text {er }}$ et $2^{\mathrm{e}}$ cycles. 


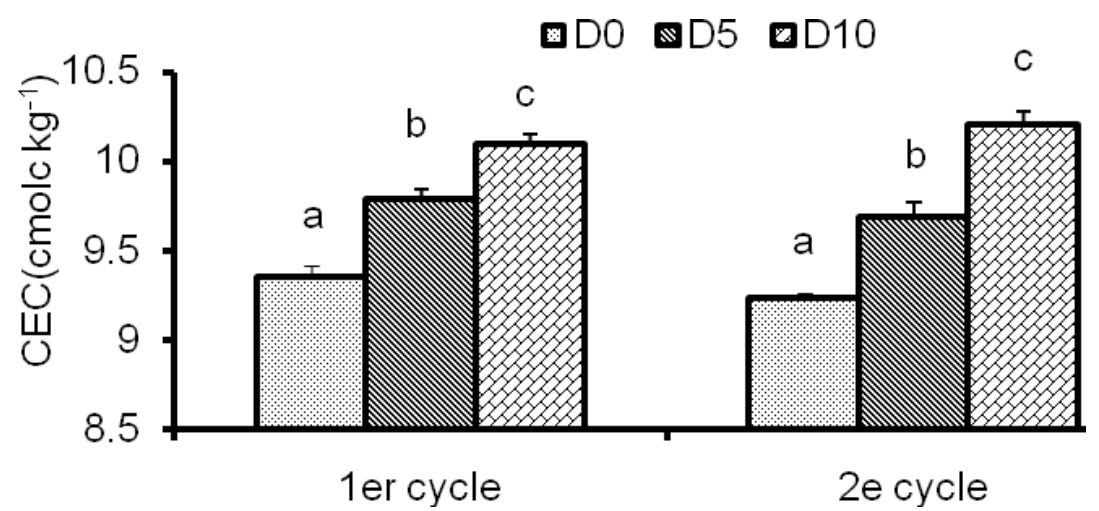

Figure 10: CEC des sols, en fonction des doses de coques, à la fin de l'essai de culture de maïs.

口D0 DD5 田D10

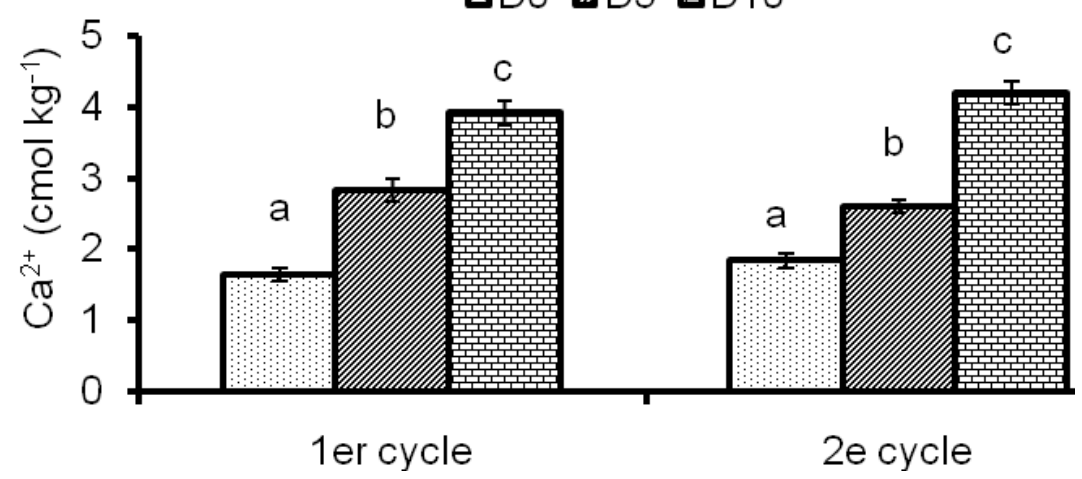

Figure 11 : Teneurs en $\mathrm{Ca}^{2+}$ des sols, en fonction des doses de coques, à la fin de l'essai de culture de maïs.

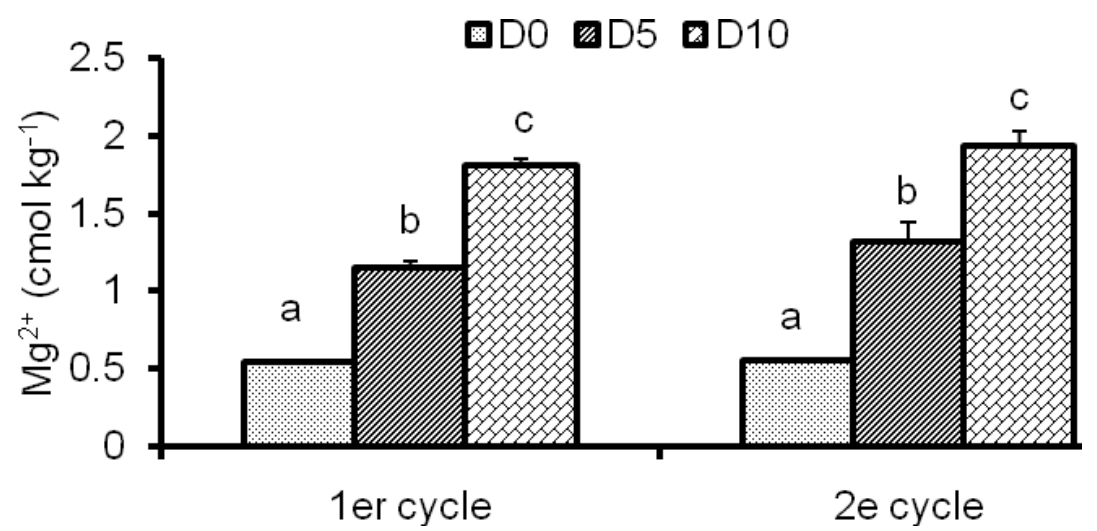

Figure 12 : Teneurs en $\mathrm{Mg}^{2+}$ des sols, en fonction des doses de coques, à la fin de l'essai de culture de maïs. 


\section{Abobi et al. J. Appl. Biosci. 2014. Influence de la fertilisation à base des coques de cacao sur les paramètres chimiques d'un ferralsol et sur la croissance du maïs à Oumé Cote d'Ivoire}

\section{田D0 四5 DD10}

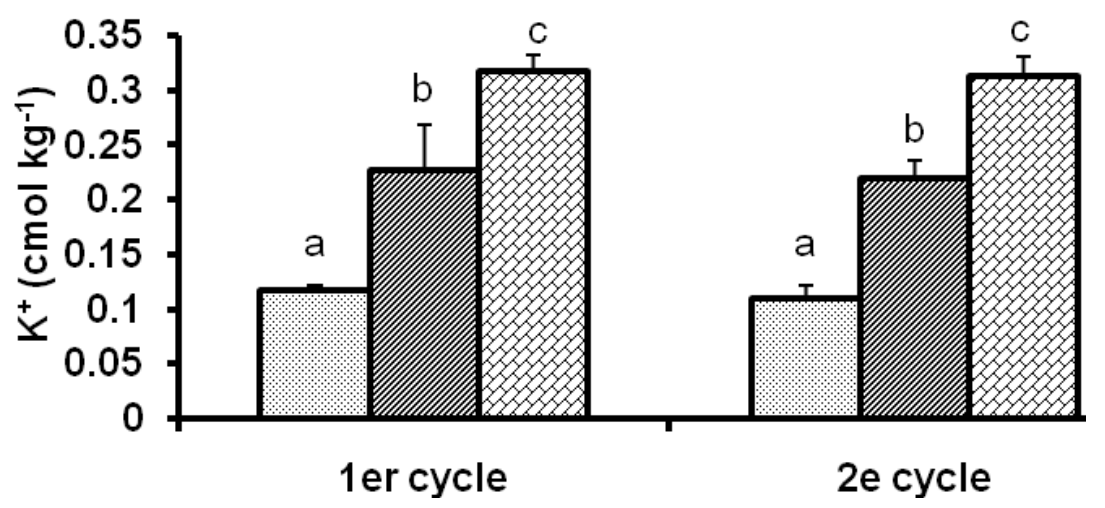

Figure 13 : Teneurs en $\mathrm{K}^{+}$des sols, en fonction des doses de coques, à la fin de l'essai de culture de maïs.

\section{DISCUSSION}

Minéralisation du carbone : L'apport des coques de cacao aux sols, à la dose D5, a occasionné une amélioration de la minéralisation du $\mathrm{C}$ organique $(64,40$ $\%)$. Cette amélioration est due à une stimulation de l'activité microbienne, liée à l'augmentation du C labile, avec l'apport des coques de cacao, matière organique, support énergétique des microorganismes (Barry, 2006). L'amélioration de la minéralisation du C organique, avec l'apport des résidus organiques, a aussi été mise en évidence dans de nombreuses études (Garcia-Gomez et al., 2003 ; Annabi, 2005). En revanche, la forte dose D10 a provoqué une baisse de la minéralisation de $\mathrm{C}$ de $70,43 \%$, par rapport à D0. Ceci est en accord avec les résultats d'Annabi (2005) et de Gamouh et al., (2005). Un facteur limitant la minéralisation du C apparaît avec la forte dose (Annabi, 2005). II pourrait être dû aux teneurs importantes de lignine, cellulose, hémicellulose et composés phénoliques lors d'un apport excessif. Leur action inhibitrice serait due à leurs propriétés antibiotiques sur les décomposeurs (Koné, 2009) et au fait que, complexées à des protéines, ces substances forment des composés difficilement accessibles aux microorganismes (Duguet, 2005 ; Koné, 2009). II existe alors un seuil au-delà duquel tout apport induit une baisse de la minéralisation de $C$.

Evolution des paramètres de croissance du maiss. Les effets positifs des coques de cacao sur la croissance du maïs ont été mis en évidence. Les valeurs des paramètres végétatifs ont été plus grandes avec la dose D5. Ces résultats corroborent ceux de Useni et al., (2013) et de Kouadio (2007); par contre, ils contredisent ceux de Djébré (2008). Ils montrent l'efficience des résidus de coques de cacao, qui peut être probablement attribuée à leur décomposition pour la mise à la disposition des éléments nutritifs. Ceux-ci favorisent la rigidité des tissus végétaux et, par conséquent, la vigueur des plantes dont les indices, obtenus avec D5, ont été, dans l'ensemble des résultats, supérieurs à ceux enregistrés avec D0, surtout au $2^{e}$ cycle. Selon FAO (2005), le potassium, à titre d'exemple, joue un rôle primordial dans le développement racinaire, dans l'absorption des cations $\left(\mathrm{Ca}^{2+}, \mathrm{Mg}^{2+}, \mathrm{NH}_{4}{ }^{+} \ldots\right)$, le maintien de la turgescence de la cellule, assurant ainsi la rigidité des tissus. L'azote et le phosphore assurent la croissance et la turgescence des plants de maïs (Paliwal, 2002). Les coques de cacao constituent donc une source d'éléments nutritifs, elles remplissent ainsi un rôle reconnu aux fertilisants organiques (Chenu, 2003). En revanche, les plants des sols fertilisés avec D10, ont présenté des valeurs inférieures à celles obtenues avec D5. Le contraire aurait été logique, car Dorn et al., (1985) ont rapporté que les doses les plus fortes fournissent plus d'éléments nutritifs pour les plantes. Nos résultats ont d'ailleurs montré qu'avec D10, les teneurs des éléments nutritifs libérés ont été les plus importantes. Mais, les biomasses enregistrées avec D10 ont été faibles, ce qui suggère que le transfert des éléments nutritifs du sol aux plants de maïs, a été moins important avec cette dose. Kouadio (2007) a aussi trouvé que les plus fortes doses ont produit un impact négatif sur la croissance des plants. II existe donc un seuil au delà duquel tout apport pourrait entraver le développement des plants, ainsi l'apport des fertilisants doit être contrôlé. Les plants des sols non fertilisés (D0) ont présenté, dans l'ensemble, les plus faibles biomasses, au terme des deux cycles de cultures. Ceci peut être attribué aux facteurs caractéristiques des sols utilisés, sols acides : pH bas 


\section{Abobi et al. J. Appl. Biosci. 2014. Influence de la fertilisation à base des coques de cacao sur les paramètres chimiques d'un ferralsol et sur la croissance du maïs à Oumé Cote d'Ivoire}

$(5,3)$, faibles teneurs en bases. Sur ces sols, l'absence d'apports de résidus de coques de cacao s'accompagne évidemment d'une perte de matières organiques et de nutriments, d'une réduction de la biomasse et de l'activité microbienne. Ce qui contribue à la baisse de la croissance et la production de la biomasse des plants de maïs. (Mulaji, 2010; Useni et al., 2013).

Evolution des paramètres chimiques du sol. Les effets significatifs des coques de cacao, sur les paramètres chimiques des sols, ont été mis en évidence par l'amélioration des CEC, du pH et des teneurs en bases, de façon proportionnelle aux doses de coques. II a été montré que l'apport de fertilisants organiques améliore les propriétés physiques, chimiques et biologiques du sol (Thyries et al., 2000, Useni et al., 2013). Les coques de cacao, à travers leur décomposition dans les sols, assurent une grande disponibilité en éléments nutritifs, même après la mise en culture. Leur utilisation dans la fertilisation des sols a été bénéfique pour la mise en culture des ferralsols pauvres de la zone d'Oumé. L'accroissement de $\mathrm{pH}$ observé dans cette étude traduit une baisse de l'acidité

\section{CONCLUSION}

L'étude a révélé que les coques de cacao ont contribué à l'amélioration de la minéralisation du C. Elles constituent une source de matière organique minéralisable. Elle a aussi montré les effets bénéfiques des résidus de coques de cacao sur les paramètres de croissance du maïs. La biodisponibilité des éléments nutritifs, par la décomposition des coques de cacao, a permis une meilleure croissance des plants de maïs, notamment avec D5. L'on est à mesure d'affirmer que l'application des résidus de coques de cacao au sol permet une amélioration de la croissance du maïs. Les apports des résidus de coques de cacao aux sols ont également permis l'amélioration des paramètres chimiques du sol, après culture du maïs, et, ce, d'autant plus que la quantité apportée a été grande. Les valeurs obtenues sur les sols fertilisés font apparaître, par rapport à celles des sols non fertilisés, des gains proportionnels aux doses de coques. Ceci

\section{REMERCIEMENTS}

Les auteurs remercient le projet "Conservation and Sustainable Management of Below Ground Biodiversity» (CSM-BGBD), pour avoir financé une du sol. L'augmentation du pH est liée aux ions $\mathrm{Ca}^{2+}$ et $\mathrm{Mg}^{2+}$, qui, en dépit de leur prélèvement par les plants, sont en quantités suffisantes pour neutraliser les ions responsables de l'acidité du sol. En effet, l'ion $\mathrm{Ca}^{2+}$, puisqu'il est antagoniste à $\mathrm{Al}^{3+}$, le déplace du complexe argilo-humique. Plus les quantités de $\mathrm{Ca}^{2+}$ et $\mathrm{Mg}^{2+}$ ont été fortes, plus la neutralisation des ions $\mathrm{H}^{+}$et $\mathrm{Al}{ }^{3+}$ a été importante et, par conséquent, l'acidité a été réduite. Ceci corrobore ce qu'a rapporté Djébré (2008) qui a indiqué que les teneurs en $\mathrm{Al}^{3+}$ avaient significativement baissé avec les doses croissantes d'incuits de chaux et de dolomie. Nos résultats ont montré une amélioration significative de la CEC, avec l'apport des coques de cacao, à chaque cycle de culture. Cette amélioration a été également rapportée par Djébré (2008), avec l'utilisation des incuits de chaux et de la dolomie. L'augmentation de la CEC pourrait être expliquée par le fait que ces amendements ont affecté les phénomènes de fixation des cations échangeables sur le complexe absorbant des sols. En effet, la nature et la teneur en cations ou en anions des fertilisants organiques apportés affectent la charge variable du sol.

nous amène à certifier que les paramètres chimiques des sols sont davantage améliorés avec l'apport de quantités croissantes des résidus de coques de cacao. Ainsi, les résidus de coques de cacao offrent des potentialités agricoles, et leur utilisation comme fertilisants organiques des sols s'avère utile pour l'amélioration de la fertilité des ferralsols. Leur intégration dans la gestion de la fertilité de ces sols dans la région du Centre-Ouest ivoirien pourrait être recommandée. La rentabilité économique de ce fertilisant organique, liée à d'autres facteurs pédoclimatiques et aux exigences des autres plantes cultivées, reste à préciser. II serait intéressant d'évaluer, dans le futur, leur impact sur les rendements des cultures, leur influence sur les paramètres physiques du sol ainsi que leurs effets sur les sols en combinaison avec les engrais minéraux.

partie de cette étude ainsi que Traoré Habib pour sa contribution dans la collecte de données. 


\section{Abobi et al. J. Appl. Biosci. 2014. Influence de la fertilisation à base des coques de cacao sur les paramètres chimiques d'un ferralsol et sur la croissance du maïs à Oumé Cote d'Ivoire}

REFERENCES BIBLIOGRAPHIQUES

Adu-Dapaah HK, Cobbina J, Asare EO, 1994. Effect of cocoa pod ash on the growth of maize. J. Agric. Sci, Cambridge, 132: 31-32.

Akanvou L, Akanvou R, Anguété K, Diarrassouba L, 2006. Bien cultiver le maïs en Côte d'Ivoire. Centre National de Recherche Agronomique. Abidjan, $4 \mathrm{p}$.

Anderson JM, and Ingram JSI, 1993. Tropical soil biology and fertility. A handbook of methods. $2^{\text {nd }} \mathrm{Ed}$. Wallingford-CAB International, 221p.

Angui PKT, Tié Bi T, Tamia JA, 2005. Typologie des sols de la Côte d'lvoire et leur utilisation. Bioterre, 5(1), 1-16.

Annabi M, 2005. Stabilisation de la structure d'un sol limoneux par des apports de composts d'origine urbaine : relation avec les caractéristiques de leur matière organique. Thèse de doctorat de l'Institut National Agronomique, Paris-Grigon, France, 280p.

Bado BV, 2002. Rôle des légumineuses sur la fertilité des sols ferrugineux tropicaux des zones guinéennes et soudaniennes du Burkina Faso. Thèse de doctorat de l'Université de Laval (Québec), 141p.

Barry Y, 2006. La transformation des apports organiques dans le sol (modèle TAO) : cas des apports riches en azote. Master Recherche CGSE : UHP, Nancy, France, 31p.

Berchoux Ch, Lecoustre R, 1986. Croissance et développement du palmier à huile: de la germination à l'entrée en production. DOC LM $\mathrm{n}^{\circ} 06$.

Boga JP, 2007. Etude expérimentale de l'impact de matériaux de termitières sur la croissance, le rendement du maïs et du riz et la fertilité des sols cultivés en savanes sub-soudaniennes, Booro-Borotou (Côte d'lvoire). Thèse de doctorat de l'Université de Cocody, Abidan. $231 \mathrm{p}$.

Boli $Z$ et Roose E. 2000. Rôle de la jachère de courte durée dans la restauration de la productivité des sols dégradés par la culture continue en savane soudanienne humide du NordCameroun. In Floret Ch. \& Pontanier R., (Eds). La jachère en Afrique tropicale. John Libbey. Paris, 149-154.

Bongoua AJ, 2002. Caractérisation de l'état de fertilité des jachères plantées en légumineuses fixatrices d'azote dans la région d'Oumé.
Mémoire de DEA, Université de Cocody, Abidjan, 59p.

Chenu C, 2003. Comment caractériser les matières organiques du sol? Fertilité du sol et systèmes de culture. Actes des Gèmes rencontres de la fertilisation raisonnée et de l'analyse de terre, 18-19 nov 2003. Paris, 107116.

Djébré LSM, 2008. Valorisation agronomique des incuits de chaux du traitement des eaux de consommation. Mémoire de DEA, Université Nangui Abrogoua, Abidjan, 54p.

Djéké DM, Angui KTP, Kouadio YJ, 2011. Décomposition des coques des cacao dans les sols ferrallitiques de la zone d'Oumé, centre-ouest de la Côte d'lvoire : effets sur les caractéristiques chimiques des sols.

Dorn CR, Reddy CS, Lamphere DN, Gaeuman JV, Lanese R, 1985. Municipal sewage sludge application on Ohio farms: Health effects. Environ, Res., 38 : 332-359.

Duguet F, 2005. Minéralisation de l'azote et du phosphore dans les sols organiques cultivés du sud-ouest du Québec. Mémoire de Maitrise, Faculté des Sciences de l'Agriculture et de l'Alimentation, Québec, 45p.

FAO, 2005. Notions de nutrition des plantes et de fertilisation des sols. Manuel de formation, Projet Promotion de l'utilisation des intrants agricoles par les Organisations de Producteurs du Niger, Rome, FAO.

FAO, 2006. World Reference Base for soil resources. World Soils Resources Report 84; Food and Agricultural Organization of United Nations, Rome Italie, $130 \mathrm{p}$.

Freijer JI, Bouten W, 1991. A comparison of field methods for measuring soil carbon dioxide evolution: experiments and simulation. Plant Soil, 135 : 133-142.

Gamouh A, Bensalah M, Abaadi N, Zyad A, Coste CM, Fournier JC, 2004-2005. Effets comparés et interactifs des pesticides et facteurs physiques sur la minéralisation de substrats carbonés dans le sol. Bull. Inst. Scientifique Sci. Vie, 26$27: 35-38$.

Garcia-Gomez A, Bernal MP, Roig A, 2003. Carbon mineralization and plant growth in soil amended with compost samples at different degrees of maturity. Waste Management \& Research, 21: 161-171. 


\section{Abobi et al. J. Appl. Biosci. 2014. Influence de la fertilisation à base des coques de cacao sur les paramètres chimiques d'un ferralsol et sur la croissance du maïs à Oumé Cote d'Ivoire}

Koné WA, 2009. Qualité des sols en zone de savane humide de Côte d'lvoire : utilisation des légumineuses herbacées comme alternative pour une valorisation des terres marginales et une agriculture durable. Thèse de doctorat : Université Nangui Abrogoua, Abidjan, 211p.

Kouadio KP, 2007. Effet du broyat de coquillages d'huître et d'escargot sur la croissance et la production du soja. Mémoire de DEA. Université Nangui Abrogoua, 33p.

Kouakou KT, 2007. Utilisation de broyat de coquille d'escargot (Achatina achatina) pour l'amendement d'un sol ferrallitique fortement désaturé et effet sur la croissance du maïs (Zea mays). DEA en Gestion et valorisation des ressources naturelles. Université Nangui Abrogoua, 36p

Koulibaly B, Traoré O, Dakuo D, Zombré PN, 2009. Effets des amendements locaux sur les rendements, les indices de nutrition et les bilans culturaux dans un système de rotation coton-maïs dans l'ouest du Burkina Faso. Biotechnol. Agron. Soc. Environ. 13(1) : 103111.

Lepoitevin C, Nuville M, Boullenger A, Maguet N, 2003. Le cacao : vers un système plus durable? Horticulture dans les pays en voie de développement. Angers, France : ENSHAP

Moyin-Jesu El, 2007. Use of plant residue for improving soil fertility, pod nutrients, root growth and pod weigth of okra (Abelmoschus esculentrum $\mathrm{L}$ ). Bioresour. Technol., 98 : 2057-2064.

Mulaji, 2010. Utilisation des composts de biodéchets ménagers pour l'amélioration de la fertilité des sols acides de la province de Kinshasa (République Démocratique du Congo). Thèse de Doctorat, université de Liège- Gembloux Agro-Biotech, 220p.

Paliwal LR, 2002. Le maïs en zone tropicale: Amélioration et production. Organisation des Nations Unies pour l'Alimentation et l'Agriculture, Rome, $381 \mathrm{p}$

Rouanet G, 1984. Le maïs. Les techniques d'agriculture tropicale. Ed. Maisonneuve et Larose, Paris, $142 \mathrm{p}$.

Somé NA, Traoré K, Traoré O, Tassembedo M, 2007. Potentiel des jachères artificielles à Andropogon spp. Dans l'amélioration des propriétés chimiques et biologiques des sols en zone soudanienne (Burkina Faso). Biotechnol. Agron. Soc. Environ. 11(3): 245252.

Thyries L, Arrufat A, Dubois M, Fellrt C, Herrmann P, Larré-Larrouy MC, Martin C, Pansu M, Remy JC, Viel M, 2000. Influence d'une fertilisation organique et de solarisation sur la productivité maraîchère et les propriétés d'un sol sableux sous abri. Étude et gestion des sols, 7 (1) : 73-88.

Useni SY, Chukiyabo KM, Tshomba KJ, Muyambo ME, Kapalanga KP, Ntumba NF, Kasangij AKP, Kyungu K, Baboy LL, Nyembo KL, Mpundu MM, 2013. Utilisation des déchets humains recyclés pour l'augmentation de la production du maïs (Zea mays L.) sur un ferralsol du sudest de la RD Congo. J. Appl. Biosci. 66:5070 5081. 